\title{
Overexpression of Agrin promotes tumor proliferation and correlates with poor prognosis in cholangiocarcinoma
}

Meimei He

wenzhou university

Shasha Ji

wenzhou university

Junxue Tu

wenzhou university

Dan Lou ( $D$ choupidan99@163.com )

Wenzhou University https://orcid.org/0000-0002-9269-4737

\section{Research}

Keywords: cholangiocarcinoma, Agrin, YAP, metastasis, proliferation

Posted Date: August 4th, 2020

DOI: https://doi.org/10.21203/rs.3.rs-50004/v1

License: (1) This work is licensed under a Creative Commons Attribution 4.0 International License.

Read Full License 


\section{Abstract \\ Background}

Agrin exists as a shorter Type II Transmembrane form with an internal signal peptide, and is closely correlated with the activation of multiple intercellular signaling pathways. The aim of the present study was to investigate the role of Agrin in the development of cholangiocarcinoma (CCA).

\section{Methods}

RT-qPCR and western blotting were performed to detect the expressional level of target genes, including Agrin, in CCA tissues or cell lines. The correlation between Agrin, and tumor characteristics and prognosis, was analyzed using independent sample t-test, the Kaplan-Meier method and Cox proportional hazard model, respectively. Proliferation, migration, invasion and tumorigenesis in CCA cells was determined by CCK8 assay, cell cycle detection, Transwell assay and nude mouse tumorigenicity assay, respectively.

\section{Results}

Agrin was significantly upregulated in CCA tissues, as compared to the adjacent non-tumor tissues, and was correlated with poorer tumor characteristics such as portal vein tumor thrombus, intrahepatic metastasis and poor survival. The Agrin overexpression in CCA cell lines clearly promoted proliferation, colony formation, migration, invasion and cell cycle progression, but Agrin knockdown had the opposite effect. Furthermore, CCA cells with inhibitory Agrin expression presented with less and smaller tumors, as compared with the control group in vivo. Mechanistic analysis indicated that Agrin was able to activate the Hippo signaling pathway and induce yap to enter the cell nucleus.

\section{Conclusions}

We found that Agrin promotes the CCA progression via activating the Hippo signaling pathway, which could be a potentially promising target for CCA treatment.

\section{Introduction}

Cholangiocarcinoma (CCA) is the most frequent biliary adenocarcinoma associated with high malignancy, accounting for $10-15 \%$ of primary liver cancer cases[1]. According to where the tumor originates from, CCA is classified into intrahepatic and extrahepatic CCA, the latter including perihilar and distal CCA. Most of CCA patients are diagnosed at a median age of 65 years, but patients with primary sclerosing cholangitis commonly present with CCA at the age of $\leq 40$ years[2]. The high incidence rates of hepatolithiasis and high prevalence of hepatitis B virus may be the main cause of CCA initiation in China[3]. In recent decades, there has been increasing concern over the rising incidence and high tumor- 
related death rates of CCA worldwide. For CCA patients, surgery is the only potentially curative option. Unfortunately, rapid preoperative tumor progression and high postoperative tumor recurrence rates significantly limit the R0 resection rates and long-term tumor-free survival (TFS) time[4]. Recently, great advances have been made in tumor management[5], and related immunotherapy has been achieved, which strongly suggests that further understanding of the molecular mechanism underlying tumor progression and recurrence would be useful for improving prognosis. The present study aimed at determining the role of Agrin in regulating cell biofunction in CCA.

Agrin is a $210-\mathrm{kDa}$ basal lamina-related heparan sulfate proteoglycan that exists as either a shorter Type II Transmembrane protein or a secreted protein in the extracellular matrix[6]. Its two parts have been shown to have a completely different function, with the carboxy-terminal part exhibiting a synaptogenic activity and triggering the acetylcholine receptors (AChRs) by activating the low-density lipoprotein receptor-related protein 4 (Lrp4) receptor and the muscle-specific kinase (MuSK), and the aminoterminal end consisting of a signal sequence, which is needed for the secretory pathway, and an aminoterminal Agrin domain, which is required for binding with basal lamina-associated laminins[7]. The majority of studies have focused on investigating the essential role of Agrin in the formation of the vertebrate neuromuscular junction during embryogenesis. For example, Nitkin et a[8] and Jones et a[9] confirmed that ectopic Agrin expression could promote the formation of postsynaptic specializations, such as the induction of endplate-specific gene transcription of the e-subunit of the AChR in vivo and aggregates of several important molecules on cultured myotubes in vitro. Conversely, the inhibition of Agrin resulted in very few pre- and postsynaptic specializations, and led to mice dying at birth due to nonfunctional respiratory musculature[10]. In addition, several recent studies have verified that Agrin is necessary for tumor initiation and progression. Sayan et al demonstrated that Agrin promoted cellular proliferation, migration and invasion in hepatocellular carcinoma, and activated the FAK signaling pathway through the formation of the Agrin-Lrp4-MuSK signaling complex[11]. Rivera C showed that Agrin silencing decreased the malignant ability, and inhibited the phosphorylation of FAK, ERK and cyclin D1 proteins in oral squamous cell carcinoma cells[12]. However, the relationship between Agrin and CCA development had not yet been explored.

Herein, it was revealed that Agrin promotes CCA malignant characteristics as an activator of the Hippo signaling pathway regulating cell proliferation, migration and invasiveness. Furthermore, shRNAmediated Agrin knockdown significantly suppressed tumor spheroid formation in vivo. Collectively, the present results showed that Agrin upregulation was required for CCA development, and that Agrin could potentially serve as a therapeutic target.

\section{Material And Methods}

\section{Cell lines and cell transfection}

Two human CCA cell lines (HUCCT-1 and CCLP-1) were used in the study, which were purchased from the Institutes of Biological Sciences (Shanghai, China) and cultured following the manufacturer's 
instructions. HUCCT-1 and CCLP-1 were incubated using 1640 complete medium (Thermo Fisher Scientific, Inc.) supplemented with $10 \%$ fetal bovine serum (FBS) and antibiotics ( $50 \mathrm{U} / \mathrm{ml}$ penicillin and $50 \mathrm{mg} / \mathrm{ml}$ streptomycin) in a $5 \% \mathrm{CO}_{2}$ incubator at $37^{\circ} \mathrm{C}$. Lentivirus encoding both an shRNA hairpin (5'CAGGAGAAUGUCUUCAAGATT-3') specifically targeting human Agrin and green fluorescent protein, as well as the vector pcDNA3.1 embracing the full-length cDNA of Agrin, were used to infect the cells, according to the manufacturer's instructions. Lentivirus encoding a scramble sequence was used as a control.

\section{RNA extraction and RT-qPCR}

The extraction of total RNA from tissues and cells was performed using TRIZOL reagent (Thermo Fisher Scientific, Inc.), following the manufacturer's instructions, and a ReverTra Ace ${ }^{\circledR}$ kit (Thermo Fisher Scientific, Inc.) was used to reverse-transcribe the RNA into cDNA. RT-qPCR was performed using SYBR Premix Ex Taq ${ }^{\text {TM }}$ (Takara Bio, Inc.) and the Thermal Cycler Dice Detection System. The following primers were used to specially amplify the Agrin gene and $\beta$-actin.

Agrin:

forward, 5'-ACACCGTCCTCAACCTGAAG-3'

reverse, 5'-CCAGGTTGTAGCTCAGTTGC-3';

$\beta$-actin:

forward, 5'-AGAGCCTCGCCTTTGCCGATCC-3'

reverse, 5'-CTGGGCCTCGTCGCCCACATA-3'.

\section{Cell cycle analysis}

Cells with Agrin plasmid or shRNA transfection received serum starvation to induce cell cycle synchronization for $48 \mathrm{~h}$, then seeded at a density of 10,000 cells per 6 -cm plate. The monolayer cells with $~ 70 \%$ confluency were harvested as single cell suspensions and fixed in $90 \%$ ethanol overnight. Following washing twice with PBS, cells were incubated with $0.5 \mathrm{ml}$ DNA Prep Stain in the dark for $30 \mathrm{~min}$ at room temperature, followed by flow cytometric analysis. The percentage of the cell population in each phase was calculated using ModFit LT software.

\section{Colony formation}

Colony formation assay was performed through seeding CCA cells in 6 wells plates. A total of 10,00 cells was seeded, and cultured in fresh medium for 2 weeks. Subsequently, the colonies stained with crystal violet were photographed and counted.

\section{Counting kit-8 assay}


The effect of Agrin on CCA cell viability was detected by cell counting kit-8 (Dojindo Molecular Technologies, Inc.) assay. Briefly, CCA cells were plated in a 96-well plate at a density of 1,000 cells per well. Following culture for 24, 48, 72, 96 and 120 h, fresh medium containing $10 \%$ Cell Counting Kit-8 was added to the well, and the absorbance value at a wavelength of $450 \mathrm{~nm}$ was detected as an indicator of cell viability.

\section{Cell migration and invasion assays}

Migration and invasion assays were conducted using the Transwell system (24-well insert, 8.0- $\mu \mathrm{m}$ pores). CCA cells of $1 \times 10^{4}$ per well were suspended in $100 \mu$ non-FBS culture medium and plated into the upper chamber. The upper chamber, which was coated with Matrigel, was used for the invasion assay, and the one without Matrigel for the migration assay. Medium containing $10 \%$ FBS was added into the bottom chamber to drive cell translocation at $500 \mu \mathrm{l}$ per well. After 24,48 and $72 \mathrm{~h}$, cells on the upper surface of the chamber were cleaned with cotton swabs, and those on the bottom surface were reserved and fixed in $95 \%$ methanol for 20 min, then stained with $0.4 \%$ crystal violet and photographed.

\section{Wound scratch assay}

CRC cells with Agrin knockdown or overexpression were respectively plated in the 6-well plate. When the monolayer CRC cells reached $80-90 \%$ confluence, a line of wound was scratched using culture-insert (Ibidi, Germany). Following incubation for 24 and $48 \mathrm{~h}$, the migrated distances were recorded and considered an indicator of migration.

\section{Western blotting}

Total protein was extracted from CAA cells using RIPA lysis buffer (Thermo Fisher Scientific, Inc.) supplemented with protease and phosphatase inhibitors (Thermo Fisher Scientific, Inc.) on ice. The electrophoretic separation of proteins was performed in $4-20 \%$ gradient sodium dodecyl sulfate-polyacrylamide gel (GenScript). The protein on the gel was then transferred to 0.45- $\mu \mathrm{m}$ PVDF membranes (EMD Millipore). Following blocking with Tris-buffered saline with $5 \%$ non-fat milk and $0.1 \%$ Tween-20 for $1 \mathrm{~h}$, the membrane was incubated with target primary antibodies at room temperature overnight at $4^{\circ} \mathrm{C}$ The secondary antibody was incubated for $1 \mathrm{~h}$ at room temperature at a dilution of 1:5,000. Protein expression was determined by Super Signal West Pico Chemiluminescent Substrate (Pierce).

\section{Tumor xenograft experiment}

A total of $1 \times 10^{6} / 0.1 \mathrm{ml}$ cells with Agrin knockdown or vector transfection as the control were subcutaneously injected into the lateral flank of 6 -week-old male BALB/c nude mice ( $n=5$ per group). Tumor volume was measured weekly with a caliper according to the following equation: Tumor size $=$ (Width ${ }^{2} x$ Length)/2. Four weeks after the injection, all mice were sacrificed and tumors were harvested.

\section{Statistical analysis}

All experiments were performed thrice and related data are presented as the mean \pm standard deviation or frequency. The difference between experimental groups was statistically analyzed using Student's $t$ and 
Fisher's exact tests. The value of CA199 was beyond $12000 \mathrm{kU} / \mathrm{L}$ (the maximum value in clinical test) in some patients, so CA199 was analyzed with chi-square test. Statistical analysis was conducted using SPSS 19.0 software (IBM Corp.), and $\mathrm{P}<0.05$ was considered to indicate a statistically significant difference.

\section{Results}

\section{Pattern and significance of Agrin expression in CCA patients}

To explore the potential effect of Agrin on CCA, the mRNA level of Agrin was detected using RT-qPCR in 162 paired CCA specimens, and it was found that Agrin was significantly upregulated in CCA tissues, as compared with the adjacent non-tumorous tissues (Fig. 1A). According to the median Agrin mRNA level, the patients were divided into 2 groups. Difference in clinicopathological factors and demographic characteristics between the 2 groups are presented in Table I. Kaplan-Meier's analysis revealed that patients with a high Agrin expression presented more frequent CCA recurrence and poorer TFS outcomes following surgical resection (Fig. 1B). Furthermore, increased Agrin expression in CCA patients was correlated with portal vein tumor thrombus (Fig. 1C), tumor size (Fig. 1D) and tumor number (Fig. 1E), suggesting a stimulatory role of Agrin in CCA development. The postoperative 3-year overall survival rate was $49.5 \%$ in the low Agrin group and $13.7 \%$ in the high Agrin group, respectively. In addition, Agrin was verified by Cox's proportional hazards regression to be an independent risk factor for CCA (TFS: hazard ratio, $2.47 ; 95 \%$ confidence interval, $1.63-3.75 ; \mathrm{P}<0.001$; Table II).

\section{Agrin promotes CCA cell proliferation}

Recombinant plasmid pcDNA3.1-Agrin containing the Agrin gene was transfected into CCLP and HUCCT cells to overexpress Agrin. After verifying the effectiveness of the Agrin plasmid, cell counting kit-8 and colony formation assay were performed to explore the effect of Agrin on CCA cell proliferation. As compared with the empty plasmid-transfected control cells, CCA cells with Agrin overexpression exhibited higher viability (Fig. 2A-B). By contrast, inhibitory Agrin expression with shRNA lentiviral vector transfection significantly suppressed CCA cell growth and colony formation (Fig. 2C-D). For cell cycle analysis, Agrin overexpression facilitated the G1-S/G2 cell cycle transition in CCA cells, but Agrin knockdown reversed that (Fig. 2E-F).

\section{Knockdown of Agrin induces apoptosis in CCA cell lines}

Flow-cytometry was performed to clarify the role of Agrin suppression on apoptosis in CCA cells. It was found that the suppression of Agrin could induce apoptosis in HUCCT and CCLP cells, as determined by FITC/7AAD staining. CCLP cells with Agrin knockdown exhibited more apoptosis (11.13 \pm 0.76$)$ than negative control $(5.12 \pm 0.45, \mathrm{P}<0.05)$ or HUCCT $(12.45 \pm 0.68$ vs $3.64 .79 \pm 0.34, \mathrm{P}<0.05)$ cells (Fig. 3$)$. 


\section{Agrin enhances CCA metastasis in vitro}

Transwell and wound healing assays were used to evaluate the effect of Agrin on the migration and invasion of CCA cells in vitro. The Agrin-shRNA-transfected cells exhibited a clearly decreased number of cells on the reverse chamber side, as compared with the controls, but ectopic Agrin expression reversed that (Fig. 4A-F). Moreover, the wound healing rate at $24 \mathrm{~h}$ was significantly inhibited when Agrin was downregulated in CCA cells (Fig. 4G-I). These results suggested that Agrin enhanced CCA cell migration and invasion.

\section{Agrin increases CCA cell tumorigenesis in vivo}

The ability of cell tumorigenesis is crucial for CCA recurrence. We investigated the role of Agrin in tumor growth in nude mice. Agrin-shRNA transfected CCLP and negative control cells were subcutaneously injected into nude mice ( $n=5$ per group). All mice were sacrificed and the tumors were harvested at the end of the experiment. As compared with the control group, the Agrin inhibition group showed an apparent decrease in tumor size and weight (Fig. 5).

\section{Agrin promotes YAP and TAZ expression}

Previous data disclosed that Agrin activated Lrp4/MuSK receptor-mediated signaling pathways to maintain the integrity of cellular focal adhesion[13], a critical mediator of the Hippo signaling pathway[14]. We therefore detected the role of Agrin in regulating the Hippo signaling pathway. The western blotting results showed that the upregulation of Agrin slightly increased the phosphorylation of MOB1 in both CCA cells (Fig. 6A-B), but only slightly promoted YAP phosphorylation in CCLP cells (Fig. 6A) and not at all in HUCCT cells (Fig. 6B). However, CCA cells with Agrin overexpression exhibited an apparent increase in YAP and TAZ. As expected, Agrin knockdown significantly inhibited the expression of YAP and TAZ in CCLP (Fig. 6C) and HUCCT cells (Fig. 6D). These data further confirmed the effect of Agrin on promoting Hippo-YAP signaling activation.

\section{Discussion}

CCA is a malignant cancer with high incident rates worldwide, whose underlying molecular mechanism is yet to be clarified. Great advances have been made in CCA, but the tumor recurrence rate for CCA remains high after surgery. Therefore, developing a potentially effective approach against CCA is urgent. Recently, multiple lines of evidence have supported that Agrin plays an oncogenic role in promoting tumorigenesis[15]. Herein, the function of Agrin in CCA-related processes was investigated for the first time. The results demonstrated that ectopic Agrin expression promoted proliferation, colony formation, migration, invasion and tumorigenesis in CCA cells. The frequently increased expression of Agrin in clinical CCA specimens and its correlation with poorer tumor characteristics and higher postoperative tumor recurrence rates was also verified. Since cytomembrane proteins are the most well-known 
therapeutic targets in multiple types of malignancies, the present results suggested that targeting Agrin may be a promising strategy against CCA.

The underlying molecular mechanism of the function of Agrin was first defined in cultured myotubes, Wallace et al showed the important role of Agrin in promoting AChRs aggregation by inducing tyrosine phosphorylation of the AChRs $\beta$-subunit[16]. Several proteins act as Agrin receptor, including the receptor tyrosine kinase MuSK, which can form a primary structural scaffold for recruiting synaptic components. In addition, Agrin activated MuSK with the Z-site consisting of 8,11, or 19 amino acids to promote the organization of the AChRs and other neuromuscular junction components[17]. Another study demonstrated that Agrin administration was could potentially treat myasthenia gravis and other neuromuscular disorders by increasing the number of AChRs and enhancing the signal transduction of the neuromuscular junction[18]. These results provided solid evidence that Agrin induces the activation of the AChRs signaling pathway. Recent studies have revealed a close relationship between AChRs and tumor development. Following AChRs agonist treatment, multiple cancer cells underwent epithelialmesenchymal transition (EMT) and the metastatic ability of cells was increased through the activation of M2 muscarinic receptor (M2R); however, blocking M2R signaling reversed this phenomenon[19, 20]. It is well known that EMT is necessary for cancer cells to breach through the underlying basement membrane and extracellular matrix. However, Chakraborty et al revealed that the molecular mechanism underlying the regulation of EMT by Agrin does not work through AChRs. The study also showed that Agrin promoted invadopodia formation and activated the integrin-FAK pathway to regulate extracellular matrix degradation and drive EMT, and suggested that the biofunction of Agrin is not limited to the neuromuscular junction[13].

Furthermore, Dasgupta et al defined the role of Agrin in increasing intracellular $\mathrm{Ca}^{2+}$ concentration after $\mathrm{KCl}$ or caffeine therapy to promote the development of excitation-contraction coupling and myotube maturation[21]. Pirkmajer et al revealed that neural Agrin raised both the expression and activity of $\mathrm{Na}+/ \mathrm{K}+-$ ATPase to regulate skeletal muscle function in response to extrinsic stimuli[22]. Moll et al demonstrated that Agrin binds to a-dystroglycan and promotes the stabilization of laminin a 5 chain to attenuate dystrophic symptoms[23]. These findings suggested that the mechanism of Agrin is far from being clarified. Membrane a-dystroglycan serves as a mechanical bridge between cytoskeleton and extracellular matrix, which binds dystrophin with other associated proteins, known as the dystrophinglycoprotein complex[24], promoting cardiomyocyte differentiation and regeneration[25]. The genes of the dystrophin-glycoprotein complex were recently shown to be a target of the Hippo signaling pathway, and the inhibition of the link between the dystrophin-glycoprotein complex and the musculoskeletal system reduced Hippo signaling[26] and matrix rigidity[27]. This evidence hinted at a close correlation between Hippo signaling and Agrin. Therefore, in the present study, we investigated whether the effect of Agrin on tumorigenesis depends on the Hippo signaling pathway. After upregulating Agrin, a significantly increased YAP and decreased YAP phosphorylation was observed in CCA cells, which was reversed following Agrin-shRNA transfection. Consistent with these findings, Chakraborty et al reported that Agrin relied on the Hippo pathway to enhance oncogenic activities[28]. 
The nuclear YAP/TAZ combines multiple transcription factors to promote a series of oncogenic transcriptions and increase the malignant ability of the tumor[14]. Its function and expression level was tightly regulated by two major upstream kinases (Mst1/2 and LATS1/2), and acted as converging effectors of the Hippo pathway[29, 30]. The mechanisms through which Agrin regulates YAP activity are considered the relay of mechanosignalling from the extracellular matrix to intracellular YAP/TAZ, since Agrin is secreted and enriched in the basement membrane. Previous data has verified that extracellular matrix stiffness is one of the main stimulants for YAP travelling into the nucleus and facilitating the transcription of target genes[31]. Chakraborty built a model where extracellular matrix stiffness was manipulated with enhancing collagen matrix concentration. Using this model, they observed Agrin inhibition in cells cultured in stiff extracellular matrix reduced YAP's nuclear localization and transcriptional activity, weakened the local extracellular matrix and provided considerable contractile strength to the cancer cells[32]. The other potential mechanism through which Agrin regulated YAP activity was increasing cell spreading and cytoskeletal tension by manipulating F-actin distributional change[33].

In conclusion, the present study demonstrated that Agrin is an important promoter of the activation and coordination of proliferation, migration and invasion in CCA cells, and that Agrin overexpression might be a prognostic marker. More therapeutic strategies against CCA could be developed by targeting Agrin in the future.

\section{Declarations}

\section{Availability of data and materials}

The datasets used and/or analyzed during the present study are available from the corresponding author on reasonable request.

\section{Ethics approval and consent to participate}

This study was approved by the Ethics Committee of The Second Affiliated Hospital of Wenzhou Medical University.

\section{Consent for publication}

Not applicable.

\section{Competing Interests}

All of authors declared that no competing interest exists.

\section{Funding}




\section{Authors' Contributors}

All authors contributed to the analysis and preparation of the manuscript. Meimei He and Dan Lou conceived and designed the study; Meimei He and Shasha Ji performed the experiment and collected related data; Junxue Tu analyzed and interpreted the data; Meimei He drafted the manuscript.

\section{Acknowledgements}

Not applicable.

\section{References}

1. Siegel RL, Jemal A, Wender RC, Gansler T, Ma J, Brawley OW. An assessment of progress in cancer control. CA Cancer J Clin. 2018;68:329-39.

2. Blechacz B. Cholangiocarcinoma: Current Knowledge and New Developments. Gut Liver. 2017;11:13-26.

3. Tyson GL, El-Serag HB. Risk factors for cholangiocarcinoma. Hepatology. 2011;54:173-84.

4. Ebata T, Yokoyama Y, Igami T, Sugawara G, Takahashi Y, Nimura Y, Nagino M. Hepatopancreatoduodenectomy for cholangiocarcinoma: a single-center review of 85 consecutive patients. Ann Surg. 2012;256:297-305.

5. Ma LJ, Feng FL, Dong LQ, Zhang Z, Duan M, Liu LZ, Shi JY, Yang LX, Wang ZC, Zhang S, et al. Clinical significance of PD-1/PD-Ls gene amplification and overexpression in patients with hepatocellular carcinoma. Theranostics. 2018;8:5690-702.

6. Burgess RW, Skarnes WC, Sanes JR. Agrin isoforms with distinct amino termini: differential expression, localization, and function. J Cell Biol. 2000;151:41-52.

7. Weisgerber C, Bousquet R, Teillet F. [Laparotomy with splenectomy in Hodgkin's disease. Critical study of results in 123 patients]. Nouv Presse Med. 1975;4:1797-800.

8. Nitkin RM, Smith MA, Magill C, Fallon JR, Yao YM, Wallace BG, McMahan UJ. Identification of agrin, a synaptic organizing protein from Torpedo electric organ. J Cell Biol. 1987;105:2471-8.

9. Jones G, Meier T, Lichtsteiner M, Witzemann V, Sakmann B, Brenner HR. Induction by agrin of ectopic and functional postsynaptic-like membrane in innervated muscle. Proc Natl Acad Sci U S A. 1997;94:2654-9.

10. Gautam M, Noakes PG, Moscoso L, Rupp F, Scheller RH, Merlie JP, Sanes JR. Defective neuromuscular synaptogenesis in agrin-deficient mutant mice. Cell. 1996;85:525-35.

11. Samsudin, Williams ML. Rational use of skim milk in a complete infant formula. II. Clinical study in premature infants. Am J Clin Nutr. 1967;20:1308-11. 
12. Rivera C, Zandonadi FS, Sanchez-Romero C, Soares CD, Granato DC, Gonzalez-Arriagada WA, Paes Leme AF. Agrin has a pathological role in the progression of oral cancer. $\mathrm{Br} \mathrm{J}$ Cancer. 2018;118:1628-38.

13. Chakraborty S, Lakshmanan M, Swa HL, Chen J, Zhang X, Ong YS, Loo LS, Akincilar SC, Gunaratne J, Tergaonkar V, et al. An oncogenic role of Agrin in regulating focal adhesion integrity in hepatocellular carcinoma. Nat Commun. 2015;6:6184.

14. Yu FX, Zhao B, Guan KL. Hippo Pathway in Organ Size Control, Tissue Homeostasis, and Cancer. Cell. 2015;163:811-28.

15. Xiong WC, Mei L. Agrin to YAP in Cancer and Neuromuscular Junctions. Trends Cancer. 2017;3:2478.

16. Wallace BG, Qu Z, Huganir RL. Agrin induces phosphorylation of the nicotinic acetylcholine receptor. Neuron. 1991;6:869-78.

17. Apel ED, Glass DJ, Moscoso LM, Yancopoulos GD, Sanes JR. Rapsyn is required for MuSK signaling and recruits synaptic components to a MuSK-containing scaffold. Neuron. 1997;18:623-35.

18. Ohno K, Ohkawara B, Ito M. Agrin-LRP4-MuSK signaling as a therapeutic target for myasthenia gravis and other neuromuscular disorders. Expert Opin Ther Targets. 2017;21:949-58.

19. Zhao Q, Gu X, Zhang C, Lu Q, Chen H, Xu L. Blocking M2 muscarinic receptor signaling inhibits tumor growth and reverses epithelial-mesenchymal transition (EMT) in non-small cell lung cancer (NSCLC). Cancer Biol Ther. 2015;16:634-43.

20. Dasgupta P, Rizwani W, Pillai S, Kinkade R, Kovacs M, Rastogi S, Banerjee S, Carless M, Kim E, Coppola D, et al. Nicotine induces cell proliferation, invasion and epithelial-mesenchymal transition in a variety of human cancer cell lines. Int J Cancer. 2009;124:36-45.

21. Bandi E, Jevsek M, Mars T, Jurdana M, Formaggio E, Sciancalepore M, Fumagalli G, Grubic Z, Ruzzier F, Lorenzon P. Neural agrin controls maturation of the excitation-contraction coupling mechanism in human myotubes developing in vitro. Am J Physiol Cell Physiol. 2008;294:C66-73.

22. Pirkmajer S, Chibalin AV. Na,K-ATPase regulation in skeletal muscle. Am J Physiol Endocrinol Metab. 2016;311:E1-31.

23. Moll J, Barzaghi P, Lin S, Bezakova G, Lochmuller H, Engvall E, Muller U, Ruegg MA. An agrin minigene rescues dystrophic symptoms in a mouse model for congenital muscular dystrophy. Nature. 2001;413:302-7.

24. Henry MD, Campbell KP. Dystroglycan: an extracellular matrix receptor linked to the cytoskeleton. Curr Opin Cell Biol. 1996;8:625-31.

25. Bassat E, Mutlak YE, Genzelinakh A, Shadrin IY, Baruch Umansky K, Yifa O, Kain D, Rajchman D, Leach J, Riabov Bassat $D$, et al. The extracellular matrix protein agrin promotes heart regeneration in mice. Nature. 2017;547:179-84.

26. Morikawa Y, Zhang M, Heallen T, Leach J, Tao G, Xiao Y, Bai Y, Li W, Willerson JT, Martin JF. Actin cytoskeletal remodeling with protrusion formation is essential for heart regeneration in Hippodeficient mice. Sci Signal. 2015;8:ra41. 
27. Yahalom-Ronen $Y$, Rajchman D, Sarig R, Geiger B, Tzahor E. Reduced matrix rigidity promotes neonatal cardiomyocyte dedifferentiation, proliferation and clonal expansion. Elife 2015, 4.

28. Chakraborty S, Njah K, Pobbati AV, Lim YB, Raju A, Lakshmanan M, Tergaonkar V, Lim CT, Hong W. Agrin as a Mechanotransduction Signal Regulating YAP through the Hippo Pathway. Cell Rep. 2017;18:2464-79.

29. Moroishi T, Hayashi T, Pan WW, Fujita Y, Holt MV, Qin J, Carson DA, Guan KL. The Hippo Pathway Kinases LATS1/2 Suppress Cancer Immunity. Cell. 2016;167:1525-39.e1517.

30. Praskova M, Xia F, Avruch J. MOBKL1A/MOBKL1B phosphorylation by MST1 and MST2 inhibits cell proliferation. Curr Biol. 2008;18:311-21.

31. Dupont S, Morsut L, Aragona M, Enzo E, Giulitti S, Cordenonsi M, Zanconato F, Le Digabel J, Forcato M, Bicciato S, et al. Role of YAP/TAZ in mechanotransduction. Nature. 2011;474:179-83.

32. Chakraborty S, Hong W. Linking Extracellular Matrix Agrin to the Hippo Pathway in Liver Cancer and Beyond. Cancers (Basel) 2018, 10.

33. Elosegui-Artola A, Andreu I, Beedle AEM, Lezamiz A, Uroz M, Kosmalska AJ, Oria R, Kechagia JZ, Rico-Lastres P, Le Roux AL, et al. Force Triggers YAP Nuclear Entry by Regulating Transport across Nuclear Pores. Cell. 2017;171:1397-410 e1314.

\section{Tables}


Table 1

Correlation of Agrin expression with clinicopathological features of human ICC

\begin{tabular}{|c|c|c|c|}
\hline \multirow[t]{2}{*}{ Variables } & \multicolumn{2}{|c|}{ Tumor Agrin expression } & \multirow[t]{2}{*}{$P$ value ${ }^{a}$} \\
\hline & Low & High & \\
\hline Age: & 35 & 47 & 0.084 \\
\hline$\leq 50$ years & 46 & 34 & \\
\hline \multicolumn{4}{|l|}{$>50$ years } \\
\hline Gender: & 33 & 56 & $<0.001$ \\
\hline Male & 48 & 25 & \\
\hline \multicolumn{4}{|l|}{ Female } \\
\hline PVTT & 11 & 42 & $<0.001$ \\
\hline Tumor size & 68 & 33 & $<0.001$ \\
\hline$\leq 5 \mathrm{~cm}$ & 13 & 48 & \\
\hline \multicolumn{4}{|l|}{$>5 \mathrm{~cm}$} \\
\hline Tumor number & 27 & 41 & 0.038 \\
\hline Single & 54 & 40 & \\
\hline \multicolumn{4}{|l|}{ Multiple } \\
\hline CA199 > 37ku/L & 68 & 58 & 0.088 \\
\hline $\mathrm{CEA}>5 \mathrm{ng} / \mathrm{ml}$ & 37 & 34 & 0.751 \\
\hline GGT > $50 \mathrm{U} / \mathrm{L}$ & 64 & 56 & 0.209 \\
\hline Histopathologic grading & 31 & 20 & 0.090 \\
\hline Well + moderately & 50 & 61 & \\
\hline Poorly & & & \\
\hline
\end{tabular}


Table 2

Agrin expression in HCC is an independent predictive factor for

\begin{tabular}{|c|c|c|c|c|}
\hline \multirow[b]{2}{*}{ Variables } & \multicolumn{2}{|c|}{ Cumulative recurrence } & \multicolumn{2}{|l|}{ Overall survival } \\
\hline & $\mathrm{HR}(95 \% \mathrm{Cl})$ & $P$ value $^{a}$ & $\mathrm{HR}(95 \% \mathrm{Cl})$ & $P$ value ${ }^{a}$ \\
\hline \multicolumn{5}{|l|}{ Age (yer) } \\
\hline$\geq 50$ vs $<50$ & $0.63(0.42-0.95)$ & 0.028 & $0.84(0.54-1.31)$ & 0.436 \\
\hline \multicolumn{5}{|l|}{ Gender } \\
\hline Male vs Female & $1.42(0.98-2.07)$ & 0.065 & $1.06(0.87-1.29)$ & 0.543 \\
\hline \multicolumn{5}{|l|}{ PVTT } \\
\hline Present vs Absent & $0.98(0.68-1.41)$ & 0.895 & & \\
\hline \multicolumn{5}{|l|}{ Tumor number } \\
\hline Multiple vs Single & $0.84(0.51-1.36)$ & 0.476 & & \\
\hline \multicolumn{5}{|l|}{ Histopathologic grading } \\
\hline Well-Moderately vs Poorly & $1.66(1.07-2.59)$ & 0.025 & $1.18(0.73-1.92)$ & 0.491 \\
\hline \multicolumn{5}{|l|}{ Tumor size } \\
\hline$>5 \mathrm{~cm}$ vs $\leq 5 \mathrm{~cm}$ & $0.88(0.59-1.33)$ & 0.567 & & \\
\hline \multicolumn{5}{|l|}{ Agrin expression } \\
\hline High vs Low & $2.65(1.79-3.89)$ & $<0.001$ & $2.47(1.63-3.75)$ & $<0.001$ \\
\hline
\end{tabular}

\section{Figures}



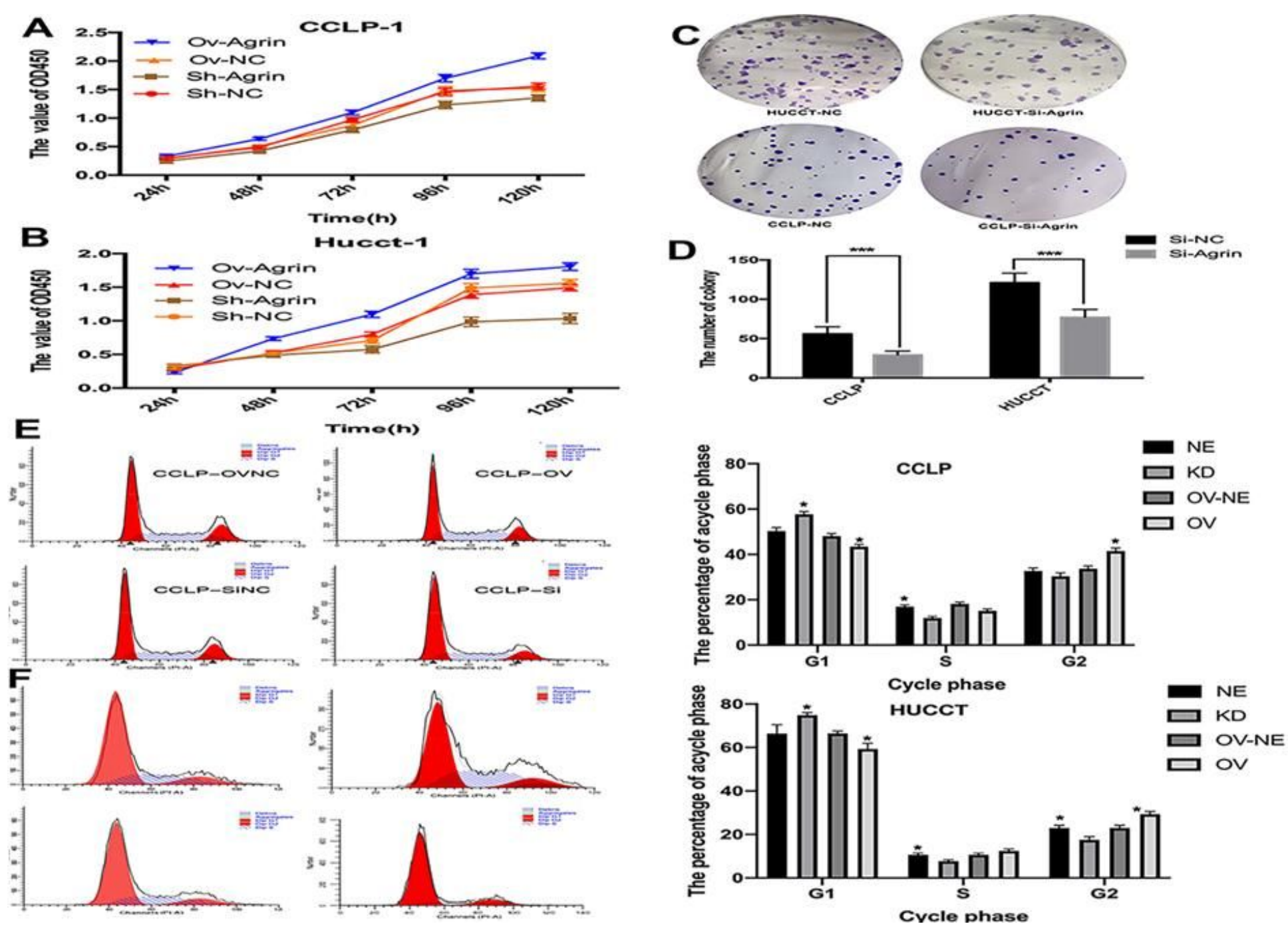

Figure 1

In 162 pair specimens, Agrin was significantly overexpressed in CCA tissues comparing to adjacent nonCCA tissues with RT-PCR (A). Furthermore, further overexpression of Agrin correlated with higher rate of death (B), PVTT (C) tumor size (D) and tumor number (E). 

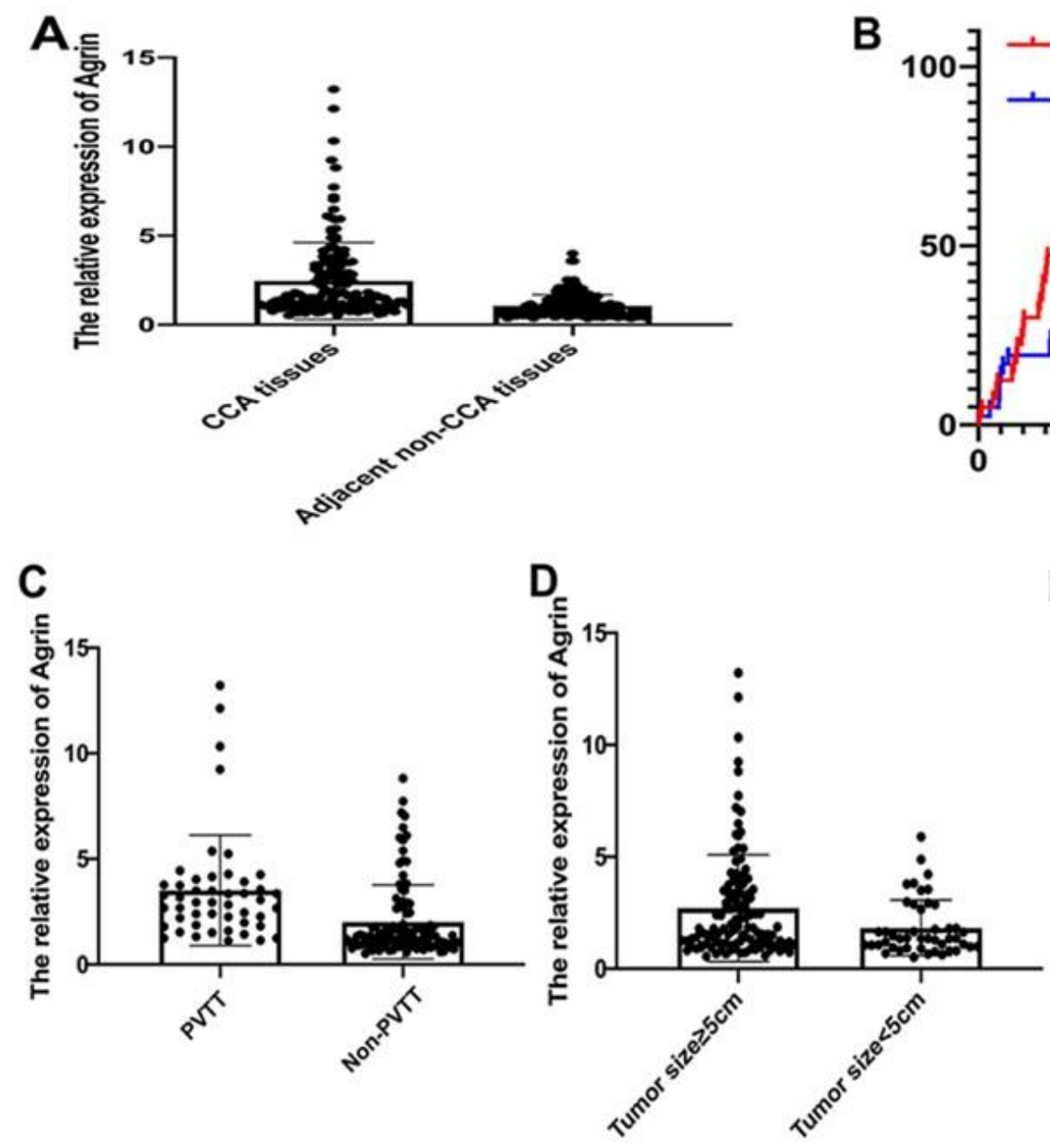

E

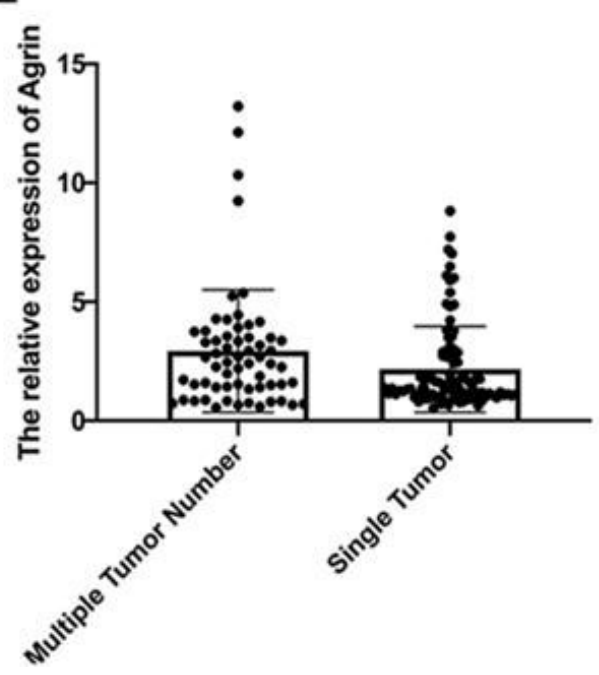

Figure 2

Comparing with the control cells, Agrin overexpression increased cell viability, but Agrin knockdown showed reverse effect in CCLP (A) and HUCCT cells (B). Agrin suppression decreased the number cell colony (C-D). Agrin overexpression facilitated the G1-S/G2 cell cycle transition, but Agrin knockdown promoted cell cycle arrest (E). F: The percentage of every cycle phase. 

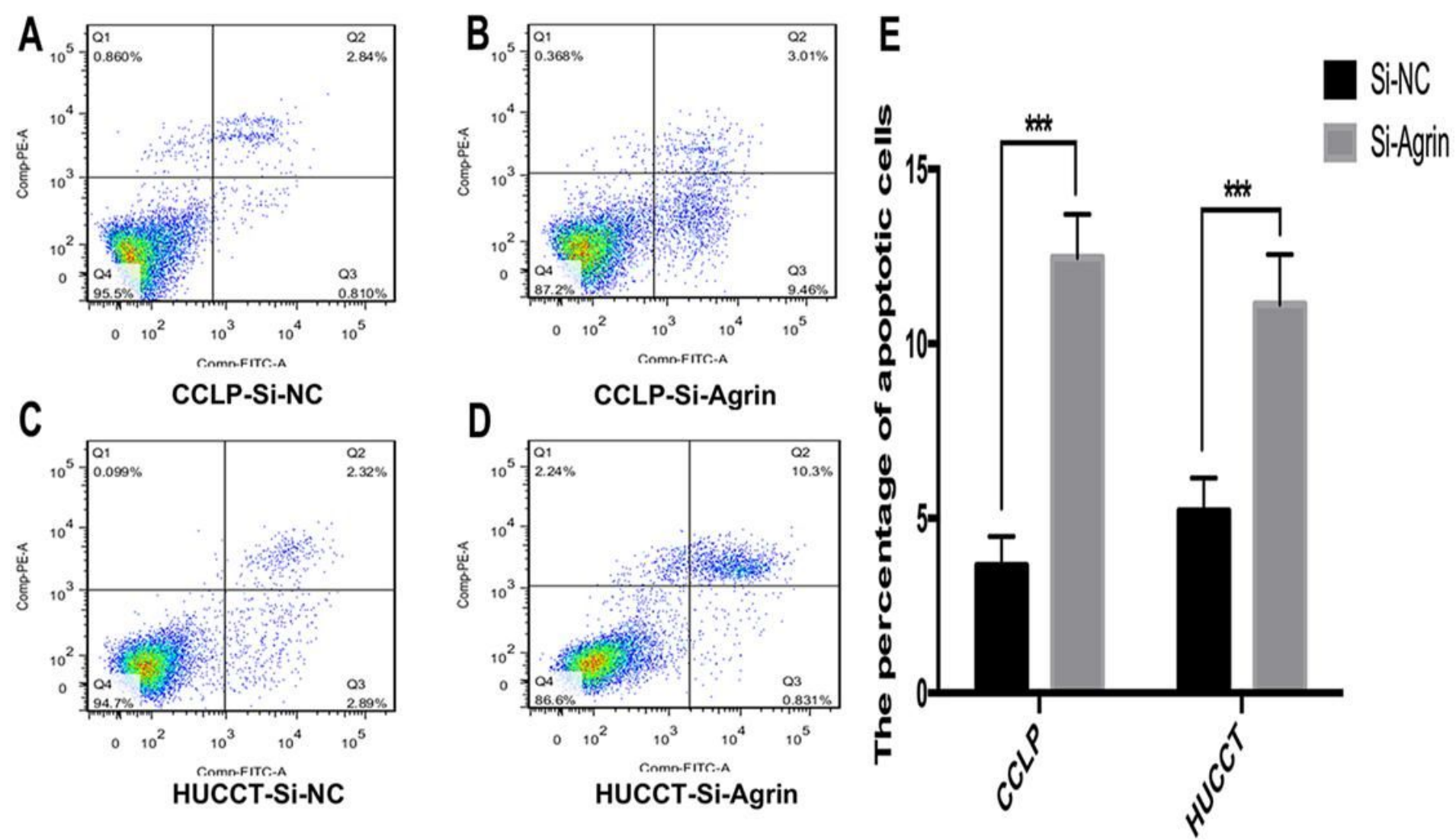

Figure 3

A flow-cytometry analysis showed CCA cells with Agrin knockdown suffered more apoptosis. A-B: CCLP cells, $11.13 \pm 0.76$ vs $5.12 \pm 0.45$, $P<0.05$. C-D: HUCCT cell, $12.45 \pm 0.68$ vs $3.64 .79 \pm 0.34$. E: The percentage of apoptotic cells. 


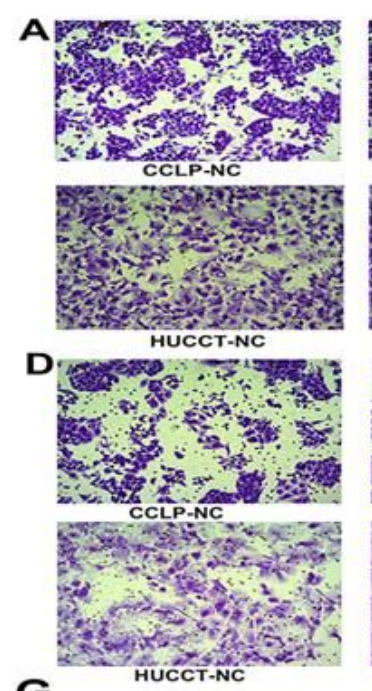

G
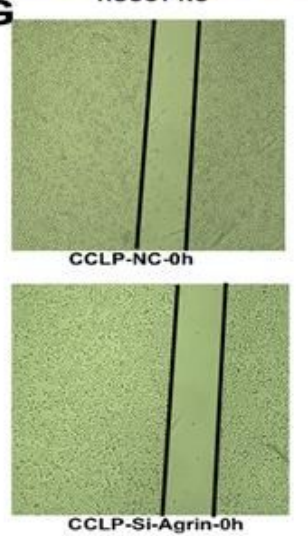

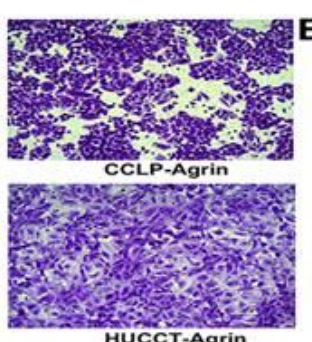

HUCCT-Agrin
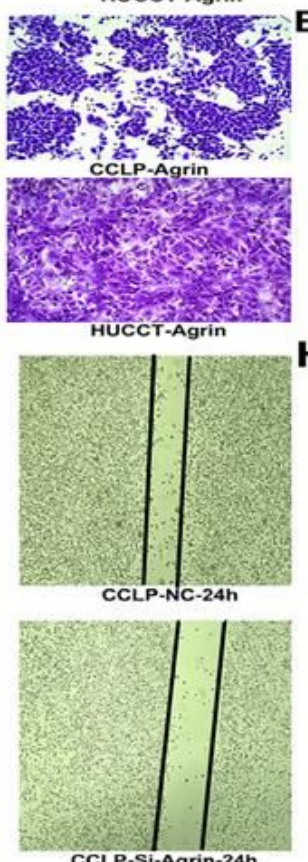

CCLP-Si-Agrin-24h

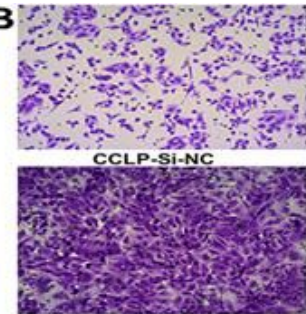

HUCCT-SI-NC
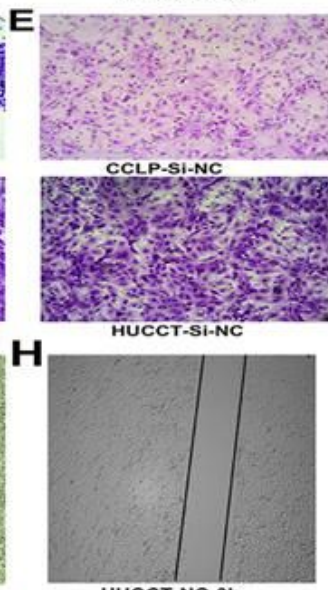

HUCCT-NC-Oh

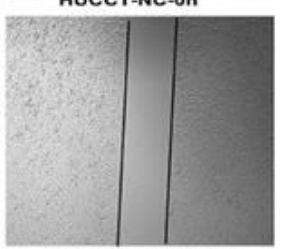

HUCCT-SI-Agrin-oh
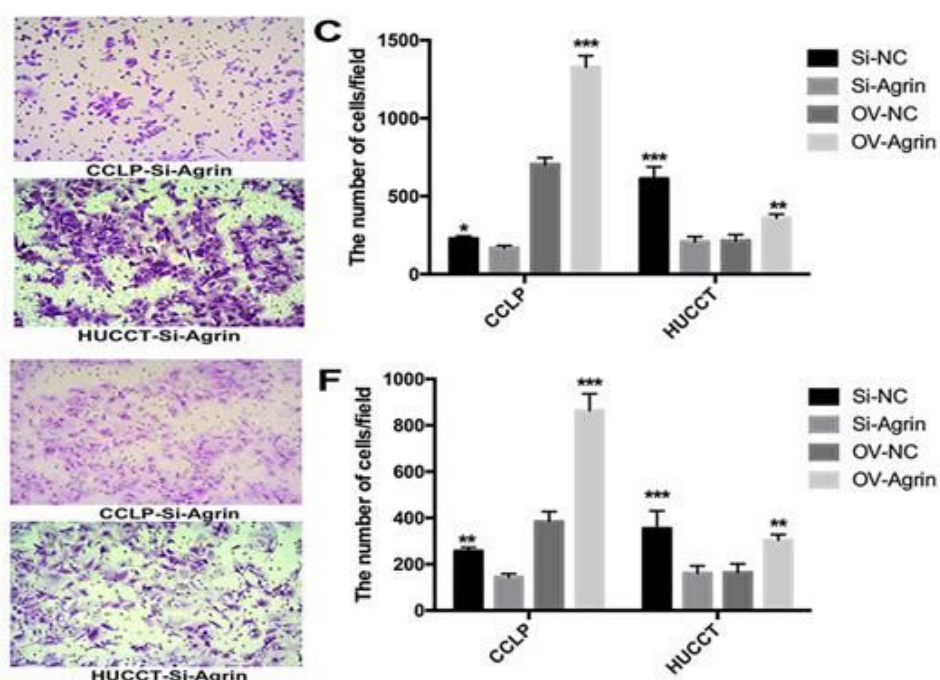

।
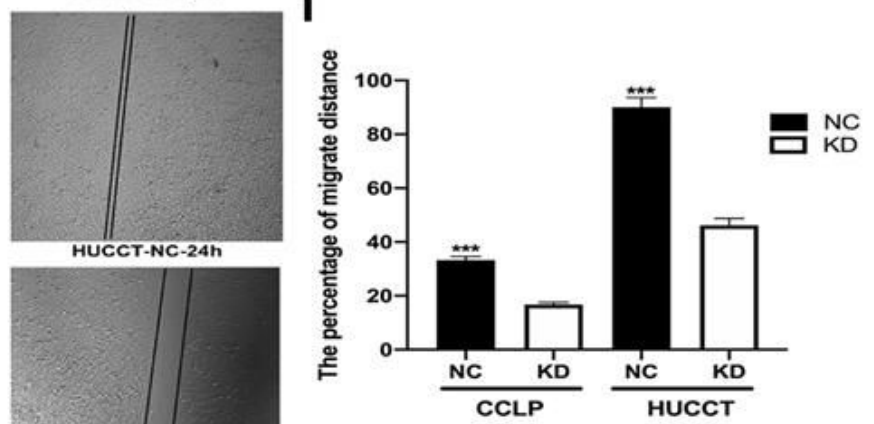

Figure 4

Ectopic Agrin expression promoted migration and invasion (A), but Agrin knockdown apparently decreased migration and invasion in CCLP (B). C: The mean cells on per field. D: Ectopic Agrin expression promoted migration and invasion in HUCCT cells. E: Agrin knockdown apparently decreased migration and invasion in HUCCT cells. F: The mean cells on per field. The wound healing rate of $24 \mathrm{~h}$ was significantly inhibited followed Agrin knockdown in CCLP (G) and HUCCT cells $(H)$. I: The percentage of migrate distance. 
A

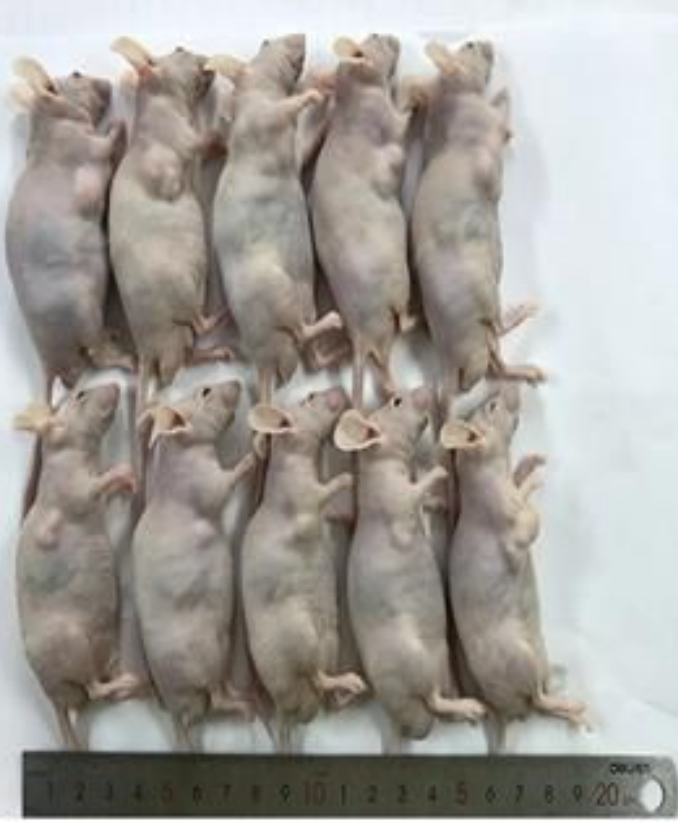

C

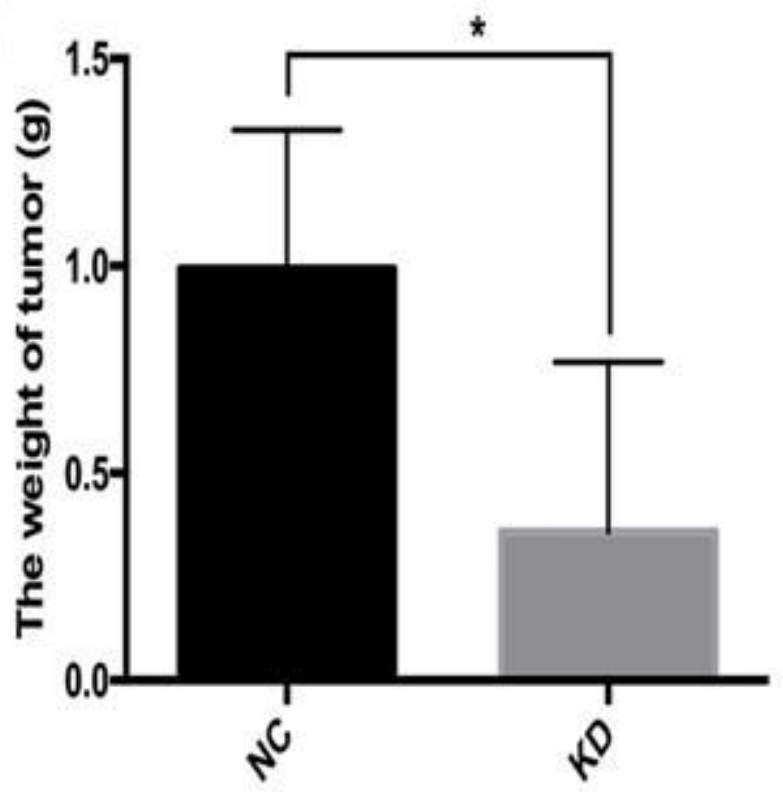

B
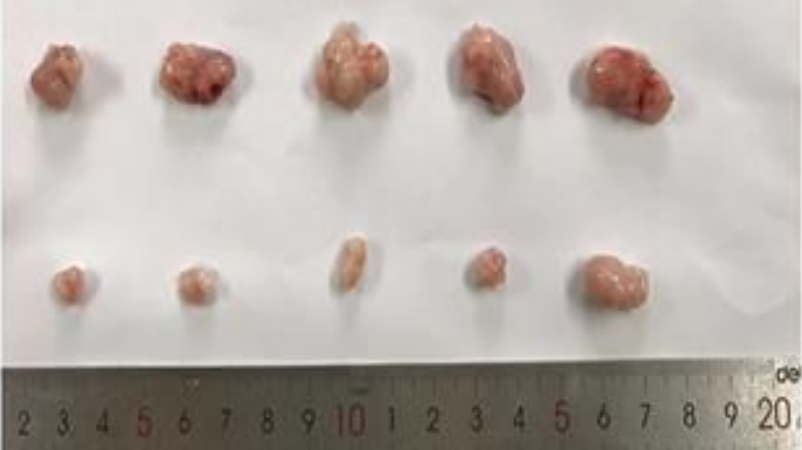

D

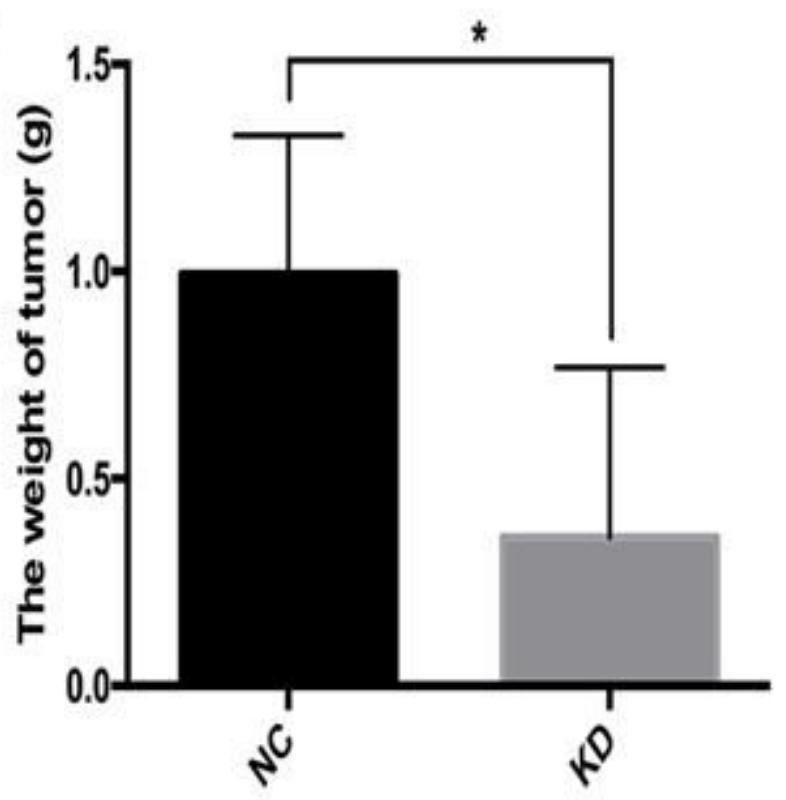

Figure 5

Comparing with the control group, CCLP cells with Agrin knockdown showed an apparently decreased ability of tumorigenesis. A: Tumor in mouse. B: Tumor separated from mouse. C: Tumor volume. D: Tumor weight. 


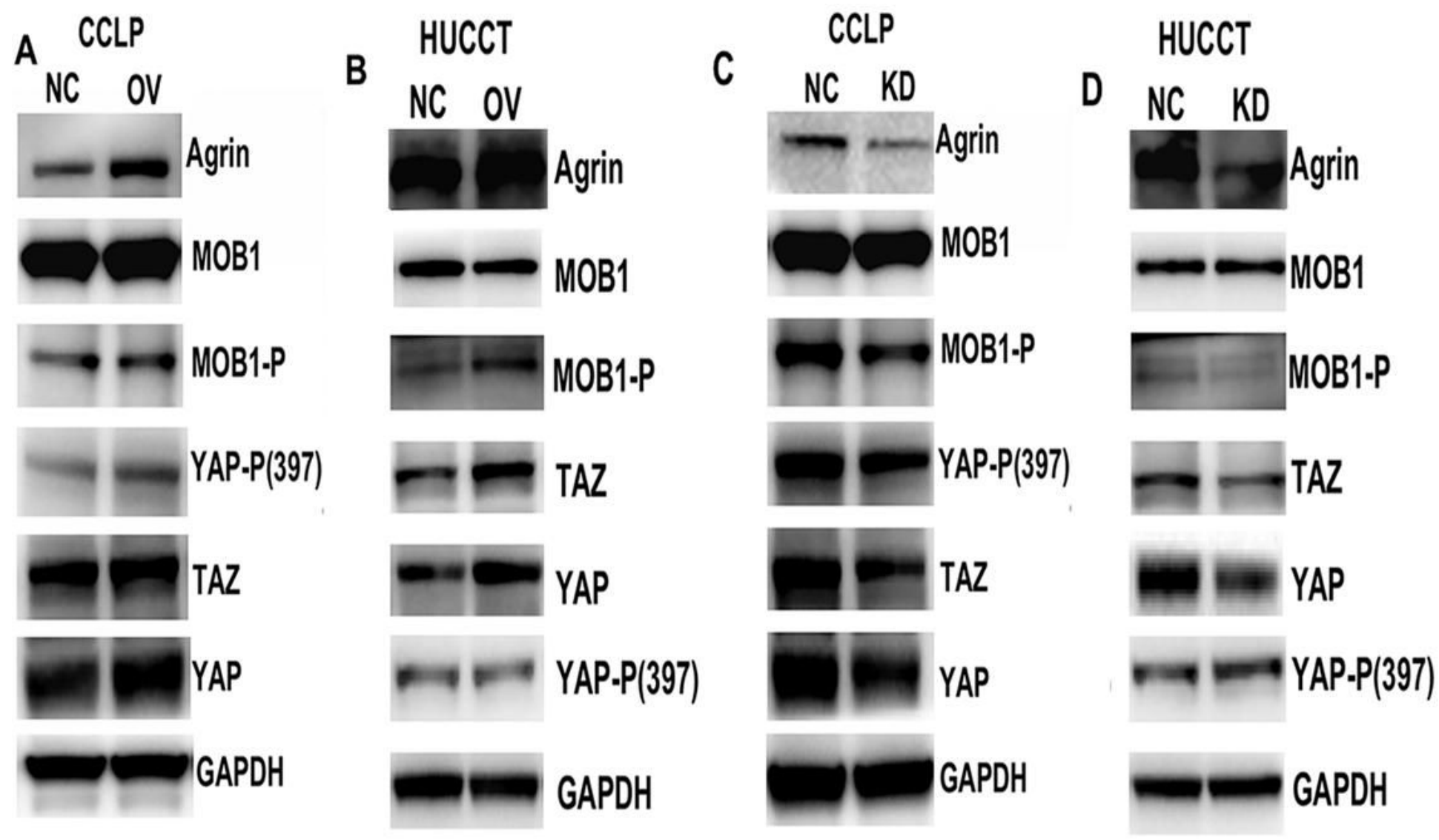

Figure 6

Up-regulated Agrin significantly increased the TAZ and YAP expression by western blotting in CCLP (A) and HUCCT cells (B). Reversely, Agrin knockdown inhibited TAZ and YAP expression in CCLP (C) and HUCCT cells (D). 